\title{
Aberrant Death Pathways in Melanoma
}

\author{
Nicholas D. Panayi, Erin E. Mendoza, \\ Elliot S. Breshears and Randy Burd \\ Additional information is available at the end of the chapter
}

\section{Introduction}

Melanoma is the most aggressive form of skin cancer and accounts for $4 \%$ of all skin cancers and nearly $75 \%$ of all melanoma deaths. According the National Cancer Institute, the incidence of melanoma is increasing 6-7\% annually. In 2012, it is estimated that there will be 76,250 new cases of melanoma and more than 9,000 deaths. Risk factors for melanoma include one or more severe sunburns, a familial history, excessive presence of moles, and fair skin. Melanoma is unique from many cancers in that it that the tumor suppressor p53 is functional in a relatively high percentage of tumors. Despite functional p53 protein, melanoma cells evade p53mediated apoptosis because of complex alterations in the apoptotic pathways. This chapter will discuss the aberrant apoptosis mechanisms that exist in melanoma and provide insight on to how these alterations could be therapeutically targeted.

\section{P53}

The tumor suppressor protein p53 has long been regarded as the guardian of the genome and is mutated in more than half of all human cancers. The functional status of $\mathrm{p} 53$ is of significant clinical importance because of its role in apoptosis, cell cycle arrest, and DNA repair; all cellular processes targeted by chemotherapy and radiation therapy [1]. The human p53 gene is highly conserved and consists of seven exons. The protein exists in a latent form and is activated through tetramerization, allowing p53 to then bind with high affinity to DNA. The tetramer conformation forms through interactions between regions in the C-terminus. Once activated, p53 can recognize sequence specific binding sites on target genes and stimulate their activation. p53 mutations are predominantly seen in the DNA binding domain, and very rarely occur in the oligomerization region [2]. The half-life of p53 is short and is increased acutely in response 
to DNA damage or changes in oxidation status. Upon activation, p53 is phosphorylated at various serine residues at the $\mathrm{N}$ or $\mathrm{C}$ terminus. The phoshorylation sites vary depending on the type of damage inflicted on the cell. Once phosphorylated, p53 acts as a transcription factor, translocating to the nucleus and binding to promoter elements of downstream target genes [3].

\section{3. p53 and apoptosis}

One of the essential roles of p53 is to maintain cellular integrity through transducing apoptosis, programmed cell death, in the event of an irreparable damage to prevent propagation of this aberrancy through uninterrupted proliferation. The mechanism of cell growth without checks and balances is the essence of malignancy; making p53 central to the governing of cell growth. Cellular damage caused by agents such as UV light, ionizing radiation, hypoxia, and oxidative stress will lead to the activation of p53, allowing for p53-dependent apoptosis to occur. The role of p53 in the mitochondrial apoptotic cascade is largely through its activities as a transcription factor for several pro-apoptotic proteins. p53 induced apoptosis can be activated via intrinsic and the extrinsic cellular pathways [4;5].

\subsection{Intrinsic apoptosis}

The intrinsic or mitchondrial apoptotic cascade results from genotoxic stress such as UV damage, oxidative stress, hypoxia, chemotherapy, or ionizing radiation. As depicted in Figure 1, activation of p53 from stress stimuli leads to activation of Bax and Bak. These proteins are important players in apoptosis and are members of the Bcl-2 family, which is made up of both pro and anti-apoptotic proteins. Family members are classified by structural similarity to the $\mathrm{Bcl}-2$ homology $(\mathrm{BH})$ domains ( $\mathrm{BH} 1, \mathrm{BH} 2, \mathrm{BH} 3$, and $\mathrm{BH} 4)$, as well as a transmembrane domain. The BH3 domain is referred to as the "death domain" and is the minimum requirement for the protein to have pro-apoptotic functions. Once activated, pro-apoptotic Bax and Bak lead to depoloarization of mitochondria and the release of cytochrome c, a small heme protein, which is bound loosely within the mitochondrial membrane. The pro-apoptotic functions of Bax and Bak are antagonized by the structurally similar anti-apoptotic proteins Bcl-2 and Bcl-xL. The $\mathrm{Bcl}-2$ family also contains ' $\mathrm{BH} 3$ only' proteins that are exclusively pro-apoptotic and include Bax, PUMA, and Bid. Upon release, cytochrome c complexes with apoptosis protease activation factor (APAF-1) to form an apoptosome. The apoptosome then binds to inactivated procaspase 9, the initiating caspase. Once activated, caspase 9 goes on to cleave caspase 3, the effector caspase responsible for executing apoptosis [6-8].

\subsection{Extrinsic apoptosis}

Extrinsic apoptosis involves death receptor activation through activation of the TNF-R family proteins (Tumor necrosis factor receptor) including Fas, PERP, and DR5. The Fas receptor is located on the cell surface and is activated by the ligand FasL, which is largely expressed by T cells. Fas ligands, which usually exist as trimmers, bind and activate their receptors by inducing receptor trimerization. Activated receptors recruit adaptor molecules such as Fas- 
associating protein with death domain (FADD), which then recruit procaspase 8 to the receptor complex, where it undergoes autocatalytic activation. Activated caspase 8 activates caspase 3 through two pathways. In the more complex pathway caspase 8 activates Bcl-2 interacting protein (Bid), which interacts with Bax to promote translocation of Bax to the inner mitochondrial membrane where it triggers cytochrome $\mathrm{c}$ release. The released cytochrome $\mathrm{c}$ binds to apoptotic protease activating factor-1 (Apaf-1). As in intrinsic apoptosis, the apoptosome leads to activation of caspase 9 , which is then able to cleave and activate procaspase 3 , ultimately leading to DNA fragmentation [8;9]. Figure 1 depicts the intersection of intrinisic and extrinsic apoptosis and onset of extrinsic apoptosis through death receptor activation.

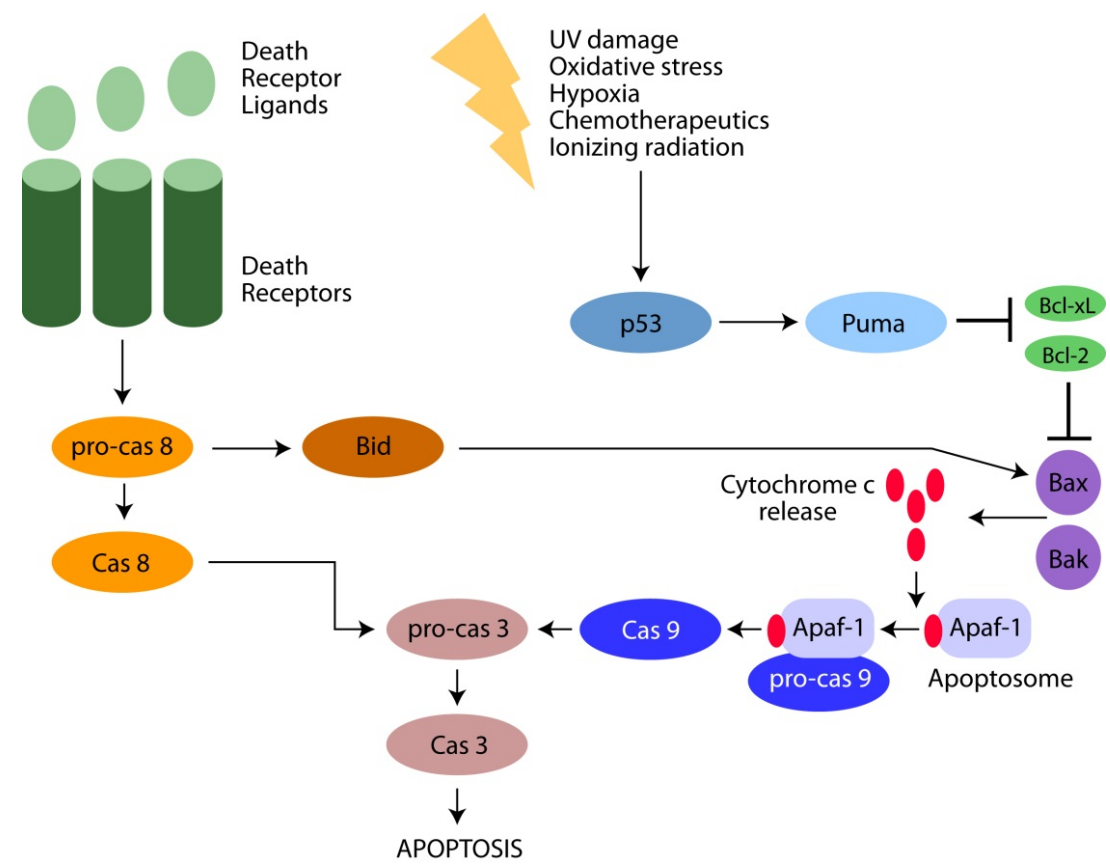

Figure 1. Intersecting intrinsic and extrinsic apoptotic pathways

\section{Role of p53 in melanogenesis}

\subsection{Tyrosinase}

The role of DNA damage and DNA repair in the stimulation of melanogenesis is corroborated by the observation that activated p53 upregulates transcription of tyrosinase, the rate-limiting enzyme involved in melanin production. Tyrosinase is a glycoprotein found in the melanosomal membrane. The protein has an inner melanosomal domain, which contains a catalytic 
region and a short transmembrane, and a cytoplasmic domain made up of about 30 amino acids. Histidine residues present in the catalytic domain portion of tyrosinase bind copper ions needed for tyrosinase activity. Structural studies of the tyrosinase promoter region have revealed the presence of a TATA box, CAAT box, 5 AP-1 sites, 2 AP-2, 2 glucocorticoid responsive elements, 2 UV responsive elements, and 3 Oct-1 sites [10]. The role of tyrosinase in pigmentation reactions is the result of the oxidation of phenols.Tyrosinase hydroxylates tyrosine in the first step of melanogenesis.Tyrosinase then catalyzes the hydroxylation of $L$ tyrosinse to $L$-Dopa and its subsequent oxidation to dopaquinone. Dopaquinone then undergoes a series of reactions that lead to the synthesis of melanin within the melanocyte. Tyrosinase has been studied as a prognostic marker of melanoma and has been useful in staging the disease and determining prognosis [11;12]. Pathological analysis of melanoma tissue samples have shown that tyrosinase detection is $97-100 \%$ specific to melanoma versus other tumor types. Detection of tyrosinase mRNA in peripheral blood in patients with metastatic melanoma using RT-PCR has been correlated to poor survival following immunotherapy with IL-2 [12]. It has also been observed that patients with primary lesions do not show tyrosinase mRNA in peripheral blood. Tyrosinase mRNA is not observed in peripheral blood in patients with previous metastatic disease but who are currently disease free, implicating that tyrosinase could be a useful prognostic tool [13].

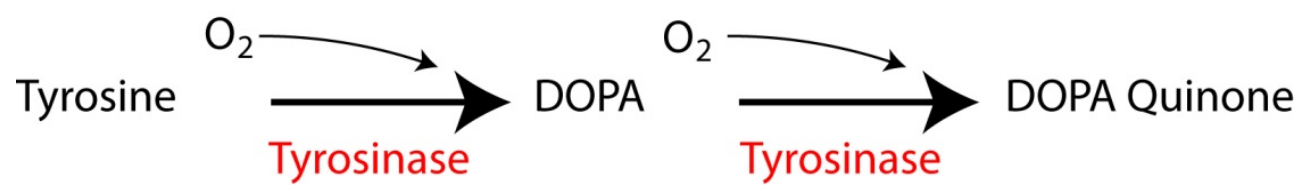

Figure 2. Tyrosinase catalyzed reactions during melanogenesis

\subsection{Melanogenesis}

The activities of p53 as a tranducer of melanin production was further elucidated by Cui et al. A p53 consensus sequence was discovered in the proopiomelanocortin (POMC) gene promoter, establishing a line of communication between UV-induced DNA damage and the sun tan response. The binding of p53 to the POMC promoter leads to an increase in the release of POMC-derived alpha-melanocyte stimulating hormone (alpha-MSH), an important messenger inducing melanogenesis. Once released, alpha-MSH acts as a ligand for the melanocortin-1 receptor (MC1R), and binding results in the production of eumelanin [14]. Yamaguchi et al. demonstrated that within keratinocytes, melanin acts as a native sunscreen. Melanin absorbs UV light, thus protecting DNA from damage. In dark skin, which possesses higher levels of melanin, protein levels of p53 are markedly lower following exposure to UV light compared to more fair skin when p53 is highly stabilized following the same exposure. The photo absorbent property of melanin protects from DNA damage, decreasing the activation of p53 [15]. Additionally, this observation supports that p53 is central to the UV damage response through stimulation of melanogenesis as an innate means to protect skin from damage through increased pigmentation. 


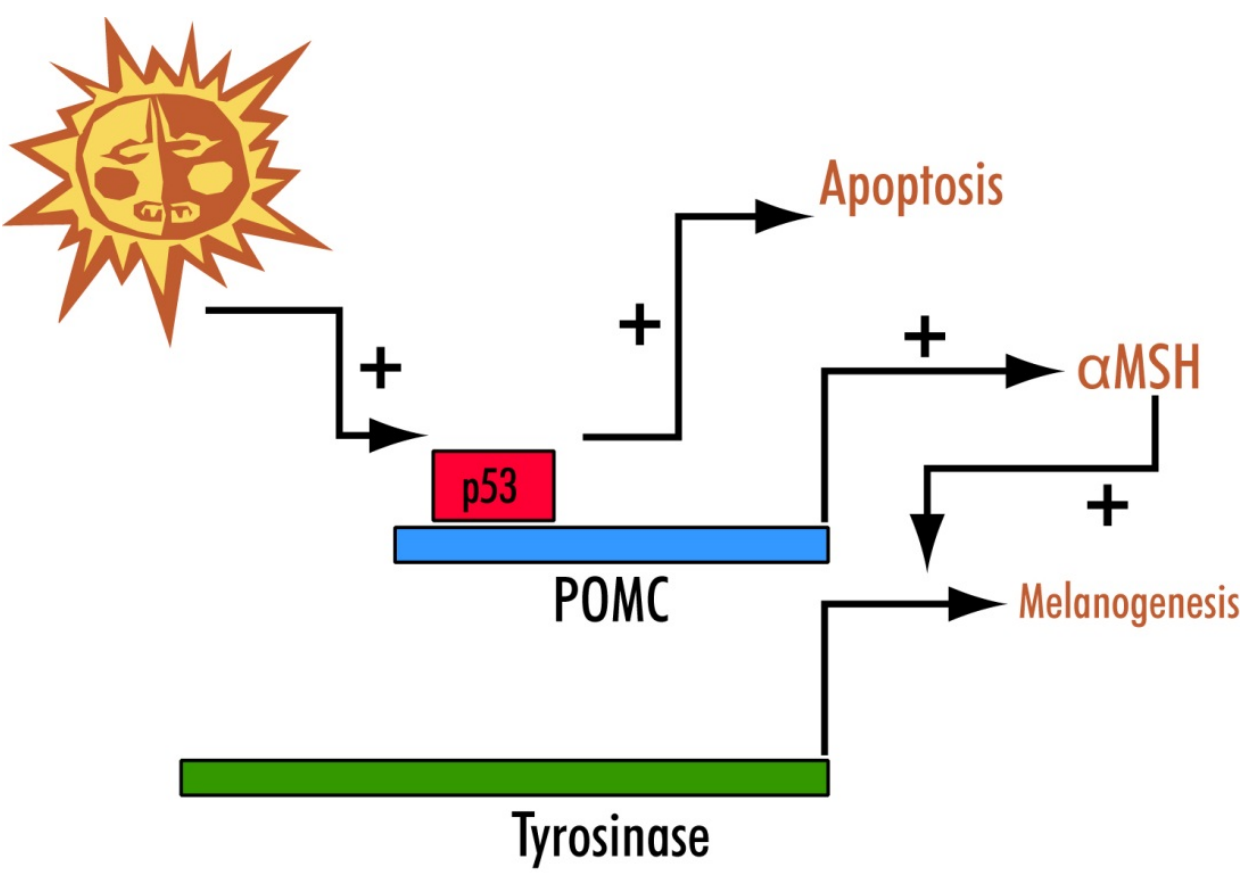

Figure 3. Role of p53 in transducing melanogenesis

\section{5. p53 in melanoma}

It has been observed that the frequency of p53 mutations in melanocytic tumors ranges from $5-25 \%$, which is considerably lower than other types of cancers. Although mutations in p53 itself remain less frequent, the functional anti-tumor properties of p53 can be repressed by many other mechanisms [16;17]. For example, p53 may be wildtype in melanoma but its repressor, MDM2, is often overexpressed, causing p53 destabilization and a decreasing in its transcriptional activities. Furthermore, the p53 upstream protein p14ARF is responsible for the regulation of MDM2; once activated, p14ARF activates p53 through inhibition of MDM2, allowing p53 to avoid proteosomal degradation and subsequent phosphorylation. Mutations or a loss of p14ARF are frequently seen in melanoma [18]. Loss of function of one or both of the p14ARF of p16INK4a proteins is thought to lead to a compensatory increase in p53 stability as melanoma progresses [19].

In normal melanocytes, the apoptotic functions of p53 are attenuated in response to UV stress in order to facilitate a longer cell life. One way this occurs is by upregulation of the p53 responsive gene GADD45a, which inhibits intrinsic apoptosis mediated through p53. It is 
speculated that this alteration in the apoptotic machinery that is specific to melanocytes could be the genesis of the inherent chemoresistence of melanoma [20].

\subsection{MDM2}

The murine double minute protein (MDM2) functions as a negative regulator of p53. Its ability to inhibit p53 is regulated by a negative feedback loop in which activated p53 leads to the transcription and translation of MDM2, which then inhibits p53 [21]. Studies conducted in mice have shown that MDM2 knockout mice are embryonic lethal in a p53-dependent manner, where incessant p53 activity causes excessive apoptosis and ultimately death. A rescue effect was observed by p53 knockout, confirming the role of MDM2 in p53 regulation [22]. MDM2 interacts with the transactivation domain of p53 via a p53-interacting domain on the $\mathrm{N}$ terminus of MDM2. This binding of MDM2 to p53 prevents p53 from binding to its transcriptional co-activators and subsequently prevents p53 from activating target genes [21]. In melanoma, MDM2 has been found to be highly expressed in half of invasive primary and metastatic melanomas. Amplification of the MDM2 locus is infrequent and over-expression is the most common occurrence. In patient follow-up studies, decreased MDM2 expression was associated with higher rates of survival. It has also been observed that both MDM2 and p53 are overexpressed in patients with melanoma [23]. This could be explained by the autoregulatory loop between p53 and MDM2. Increased expression of MDM2 is also possible due to the loss of its repressor p14 ${ }^{\mathrm{ARF}}$, a common mutation or deletion seen in melanoma [24]. Without its repressor, MDM2 is constitutively active. This could account for the apoptotic resistance observed in melanoma despite the largely wild-type status of p53.

\subsection{INK4a/ARF locus}

The INK4a/ARF locus contains the open reading frames of the proteins $\mathrm{p} 16^{\mathrm{INK} 4 \mathrm{a}}$ and $\mathrm{p} 14^{\mathrm{ARF}}$. This locus is of particular importance in melanoma because primary melanoma tumors and nearly all melanoma cell lines carry a deletion at this locus [25]. Melanocytes have an intrinsically longer life than many other cell types in the body. The anti-proliferative properties of p53 may be attenuated in melanocytes to accommodate this longer cell life. All normal cells have a finite number of cellular divisions. After this number is reached the cells enter a phase called senescence, which is a protective cellular mechanism in cancer because it prevents aberrant cell growth. Senescence can also be triggered through tumor suppressor proteins when damage is sensed, minimizing uncontrolled cell growth and halting the path to malignancy [26]. The p16 $6^{\mathrm{INK} 4 \mathrm{a}} / \mathrm{Rb}$ pathway as shown in Figure 4, is a key pathway for triggering cellular senescence. The loss of this pathway in melanoma is thought to contribute to the pathogenesis of melanoma. It is likely that loss of this protective cellular pathway is more damaging than the potential loss of p53, offering an explanation as to why a p53 mutation is not required for melanocytic tumorigenesis [26]. The $\mathrm{p} 16^{\mathrm{INK} 4 a}$ protein works as a tumor suppressor by binding to the cyclin-D-dependent protein kinases Cdk4 and Cdk6, preventing them from phosphorylating $\mathrm{Rb}$ and preventing entrance into the cell cycle. The genes that encode for both p16INK4a and Cdk4 have be identified as melanoma susceptibility genes [27]. The CdkN2A locus encodes p16INK4a as well as the tumor suppressor p14 ${ }^{\mathrm{ARF}}$. The $\mathrm{p} 14^{\mathrm{ARF}}$ gene 
is encoded by a different promoter through the use of alternative reading frames. Both p14ARF and p16I ${ }^{\mathrm{NK4a}}$ share exons 2 and 3, while Exon 1B encodes p14 ${ }^{\mathrm{ARF}}$ and Exon 1A encodes for $\mathrm{p} 16^{\text {INK4a }}$. The two share no sequence homology and are not isoforms. The main regulatory function of $\mathrm{p} 14^{\mathrm{ARF}}$ is its binding to MDM2, which prevents the ubiquitination and subsequent degradation of p53 [28]. In cases of familial melanoma, either p16INK4a or both p16 ${ }^{\mathrm{NK} 4 \mathrm{a}}$ and $\mathrm{p} 14^{\mathrm{ARF}}$ are mutated or deleted. It is rare to see mutations or deletions of $\mathrm{p} 14 \mathrm{ARF}$ alone. Germline mutations at the $\mathrm{p} 16^{\mathrm{INK} 4 a} / \mathrm{p} 14^{\mathrm{ARF}}$ locus are observed in $20-40 \%$ of familial cases of melanoma [25]. Somatic melanomas often involve mutations, deletions, or methylation of p16INK4a. Due to a shared open reading frame, up to $40 \%$ of mutations or deletions that affect p $16^{\text {INK4a }}$ will affect $\mathrm{p} 14^{\mathrm{ARF}}$ [29]. Loss of homozygousity of the $\mathrm{p} 16^{\mathrm{INK} 4 a} / \mathrm{p} 14^{\mathrm{ARF}}$ locus is observed in half of sporadic melanomas and loss of $\mathrm{p} 16^{\mathrm{INK} 4 \mathrm{a}}$ and $\mathrm{p} 14^{\mathrm{ARF}}$ expression occurs in 50-70\% of invasive melanomas [30].
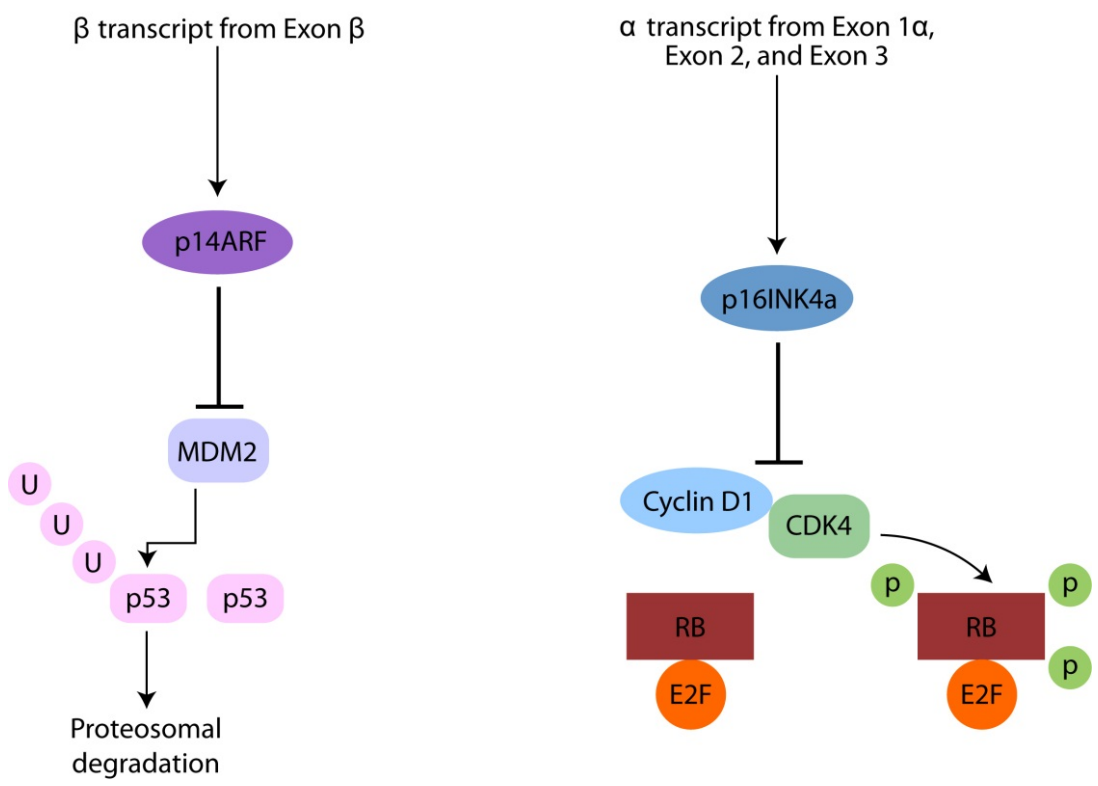

Figure 4. Gene products from the INK4a/ARF locus

\subsection{APAF-1}

Apoptosis protease-activating factor-1 (APAF-1) is a regulator in the intrinsic apoptosis pathway. When APAF-1 complexes with cytochome c a multimeric apoptosome is formed that recruits and activates pro-caspase 9 , the initiator of the mitochondrial apoptotic cascade. Once activated, caspase 9 proceeds to activate pro-caspase 3 for the execution of apoptosis. A p53 transactivation site has been identified on APAF- 1 determining that APAF- 1 is a p53 responsive gene [31]. Downregulation of APAF-1 has been observed at the protein and mRNA level 
during melanoma progression, possibly contributing to the ability of melanoma to evade apoptosis and thus conferring chemoresistance. Noncancerous melanocytes have normal APAF-1 protein and mRNA levels. Conversely, loss of homozygousity has been detected in $42 \%$ of metastatic melanoma samples. Analysis of 19 metastatic lines revealed 10 lines with low APAF-1 staining. Other studies have demonstrated significantly higher APAF-1 staining in benign nevi compared with primary melanomas. Lower APAF-1 staining has also been associated with greater tumor thickness in primary melanocytic tumors [32;33].

\subsection{Bcl-2}

The proapoptotic namesake for the Bcl-2 protein family has a complicated role in melanocytes and in melanoma. Its proapoptotic role in melanocytes prevents premature cell death to allow for continued production of pigment during the long life of the melanocyte. Bcl-2 knockout studies in mice resulted in greying of the mice two weeks after birth due to melanocyte death. The prognostic role of $\mathrm{Bcl}-2$ in melanocytic tumors is controversial. Some analyses have shown that melanoma primary tumors overexpressing Bcl-2 have an increased risk for invasion and are responsible for drug resistance. It has also been observed that Bcl-2 levels actually decrease in melanoma progression. Tumorigenic melanoma cells may take advantage of high endogenous Bcl-2 levels to survive under adverse environmental conditions that they may encounter during metastatic transformation and chemotherapeutic intervention $[5 ; 34]$.

\subsection{Bax}

The protein Bcl-2 associated X protein (Bax) is a pro-apoptotic Bcl-2 family member highly involved in the mitochondrial apoptotic cascade. It is a p53 target gene and in response to stress, p53 will bind to the Bax promoter leading to its transcription, making it a valuable indicator of p53 transcriptional activity as well as an indicator of intrinsic apoptosis. Bax will translocate to the inner mitochondrial membrane, facilitating the depolarization and release of cytochrome c. It has been observed that expression of Bax increases with melanoma progression, although the tumors remain largely chemoresistant. This implicates that a ratio effect is taking place; the inherent increase of Bcl-2 in melanocytes is potentially high enough to negate the increased expression of Bax, thus facilitating tumor resistance to apoptosis. An increase in Bax expression could also be a result of increased p53 stability and transcriptional activity from mutation or deletion of proteins at the INK4a/ARF locus [35;36].

\subsection{PUMA}

The BH3-only mitchondrial protein p53 upregulated modulator of apoptosis (PUMA) belongs to the Bcl-2 family of apoptotic regulators. The BH3 domain and its localization within the mitochondria are necessary for PUMA to induce apoptosis or decrease cell growth. Studies have not demonstrated that PUMA is post-translationally modified like other $\mathrm{BH} 3$ only proteins. In response to stress stimuli, PUMA is transactivated by p53 [37]. Once activated, PUMA promotes the apoptotic pathway through inhibition of the anti-apoptotic protein Bcl-2, allowing Bax and Bak to become activated and fosters cytochrome $c$ release from the inner mitochondrial membrane to signal initiation of apoptosis. The main pro-apoptotic effect of PUMA is such 
thatonceactivated by $\mathrm{p} 53$, it willinhibitBcl-2anti-apoptoticfamily membersinordertotransduce apoptosis [38]. It is possible that a loss of PUMA activity in melanoma cells could give rise to an increase in Bcl-2 activity, leading to either evasion of apoptosis by therapy or propagating transformation [39]. Complete silencing of PUMA in melanoma is not known. However, immunostaining for PUMA in benign nevi have shown increased PUMA staining in comparison to dysplastic nevi, primary melanoma, and metastatic tissues. When this data was compared to patient 5-year survival, patients exhibiting increased PUMA staining had a better prognosis and therapeutic response compared to those with little or no PUMA staining [40].

\section{7. p73}

The p73 protein is a p53 homolog with tumor supressor activities, but it can also demonstrate oncogenic properties, as the p73 gene can be transcribed into several different isoforms. The full length p73 (TAp73) contains a N-terminal transactivation domain, proline rich region, DNA binding domain, and C-terminal oligmerization domain. Members of the p53 family generally carry approximately 70\% sequence homology to p53, primarily in the DNA binding domain. Homology in the DNA binding domain allows p73 to recognize and regulate p53 target genes. Splice variants are generated through the use of an internal promoter, the use of an alternative translation start site, or alternative splicing of the first exons. The splice variants have a truncated N-terminus and do not contain a functional DNA binding domain, resulting in a dominant negative protein with anti-apoptotic and growth promoting characteristics. These variants are referred to as delta Np73. The delta isoforms possess antagonistic properties and counteract the tumor supressor activities of TAp73.The intracellular concentration of the of the delta isoforms in relation to the concentration of TAp73 is believed to be of significance. The ratio of the delta isoforms to TAp73 levels may determine the role p73 plays in cell survival [41;42]. Loss or mutation of the p73 gene have not been determined to be major events in the development in melanoma. However, immunostaining of several of the Delta isoforms have shown increased levels of delta isoforms in metastatic melanoma, in addition to strong p73 staining. This suggests p73 may have a role as a positive regulator of tumor growth. In this situation, the ratio of the delta isoforms to TAp73 may be of importance [43]. It is important to note that the delta isoforms are markedly more stable in comparison to TA73, promoting the antagonistic effects. It has been observed that MDM2 can disrupt p73 activities through interfering with the acetyl-transferase p300/CBP. The interaction between p53 and p73 with MDM2 are similar in that they both interact with the same hydrophobic pocket of MDM2, suggests that increased MDM2 activity may decrease the actions of TAp73 as well as that of its isoforms [44].

\section{6. p53-related targeted therapy}

Targeting p53 pathways pharmacologically can serve as a very effective means of circumventing chemoresistance observed in melanoma. The use of the small molecule inhibitor nutlin-3 to inhibit MDM2 can restore the anti-tumor effects of p53. Due to the common loss of p14ARF seen in melanoma and the compensatory increase in MDM2, p53 is unable to serve as a mediator of chemosensitivity. The sequesteration of MDM2 in combination with temezo- 
lomide potentiated the effects of the drug and increased sensitivity in vivo. The use of nutlin-3 to reinstate p53 function was also shown to be successful with the topoisomerase inhibitor Topotecan [45]. It has also been suggested that the use of nutlin-3 could reverse inaction of p73 through MDM2 inactivation. Given the presence of antagonistic isoforms of p73, the presence of MDM2 could potentially be a defect to exploit in melanoma in order to cease the antiapoptotic functions of the delta isoforms. Another possibility of therapeutic intervention is the combination of $\mathrm{p} 73$ antissense in order to reduce the antagonistic effects of $p 73$ while restoring the native function of $\mathrm{p} 53$.

Along with the loss of the $\mathrm{p} 16^{\mathrm{INK} 4 a} / \mathrm{p} 14^{\mathrm{ARF}}$ locus, an overexpression of cyclin D1 can occur in melanoma, exacerbating the lack of the G1/S cell cycle checkpoint. Sauter et. al. demonstrated that cyclin D1 antisense can work to sensitize melanoma cell lines to apoptosis. The intrinsic increase of Bcl-2 in melanoma results in apoptotic resistance and a decrease in therapeutic outcomes [46]. A study of 771 patients with advanced melanoma were treated with the Bcl-2 antisense Oblimersen in combination with Dacarbazine and had improved 24-month survival and drug response, indicating a role of $\mathrm{Bcl}-2$ in chemoresistence [47].

\section{Conclusion}

P53-mediated signaling in melanoma provides exceptional insight into the delicate signaling interplay involved in melanoma progression. The role of p53 in the compensatory responses to the infamous deletions and mutations associated with melanocytic tumors will further elucidate mechanisms involved in tumor progression. Increased understanding of these pathways will no doubt propel development of new treatment protocols forward, resulting in new and more effective means to treat this very aggressive disease.

\section{Author details}

Nicholas D. Panayi ${ }^{1}$, Erin E. Mendoza ${ }^{2}$, Elliot S. Breshears ${ }^{2}$ and Randy Burd ${ }^{2 *}$

*Address all correspondence to: rburd@u.arizona.edu

1 College of Medicine, University of Arizona, Tucson, AZ, USA

2 Department of Nutritional Sciences, University of Arizona, Tucson, AZ, USA

\section{References}

[1] Chene P. Targeting p53 in cancer. 2001 Aug. 
[2] Chene P. The role of tetramerization in p53 function. Oncogene 2001 May 10;20(21): 2611-7.

[3] Gu B, Zhu WG. Surf the post-translational modification network of p53 regulation. Int J Biol Sci 2012;8(5):672-84.

[4] Haupt S, Berger M, Goldberg Z, Haupt Y. Apoptosis - the p53 network. J Cell Sci 2003 Oct 15;116(Pt 20):4077-85.

[5] Hussein MR, Haemel AK, Wood GS. p53-related pathways and the molecular pathogenesis of melanoma. Eur J Cancer Prev 2003 Apr;12(2):93-100.

[6] Shamas-Din A, Brahmbhatt H, Leber B, Andrews DW. BH3-only proteins: Orchestrators of apoptosis. Biochim Biophys Acta 2011 Apr;1813(4):508-20.

[7] Lindsay J, Esposti MD, Gilmore AP. Bcl-2 proteins and mitochondria--specificity in membrane targeting for death. Biochim Biophys Acta 2011 Apr;1813(4):532-9.

[8] Sayers TJ. Targeting the extrinsic apoptosis signaling pathway for cancer therapy. Cancer Immunol Immunother 2011 Aug;60(8):1173-80.

[9] Mackenzie SH, Clark AC. Targeting cell death in tumors by activating caspases. Curr Cancer Drug Targets 2008 Mar;8(2):98-109.

[10] Khlgatian MK, Hadshiew IM, Asawanonda P, Yaar M, Eller MS, Fujita M, et al. Tyrosinase gene expression is regulated by p53. J Invest Dermatol 2002 Jan;118(1):126-32.

[11] Gradilone A, Cigna E, Agliano AM, Frati L. Tyrosinase expression as a molecular marker for investigating the presence of circulating tumor cells in melanoma patients. Curr Cancer Drug Targets 2010 Aug;10(5):529-38.

[12] Mellado B, Del C, V, Colomer D, Gutierrez L, Castel T, Quinto L, et al. Tyrosinase mRNA in blood of patients with melanoma treated with adjuvant interferon. J Clin Oncol 2002 Oct 1;20(19):4032-9.

[13] Visus C, Andres R, Mayordomo JI, Martinez-Lorenzo MJ, Murillo L, Saez-Gutierrez $\mathrm{B}$, et al. Prognostic role of circulating melanoma cells detected by reverse transcriptase-polymerase chain reaction for tyrosinase mRNA in patients with melanoma. Melanoma Res 2007 Apr;17(2):83-9.

[14] Cui R. Central role of p53 in the suntan response and pathologic hyperpigmentation. 2007 Mar 9.

[15] Yamaguchi Y, Brenner M, Hearing VJ. The regulation of skin pigmentation. J Biol Chem 2007 Sep 21;282(38):27557-61.

[16] Benjamin CL. p53 and the pathogenesis of skin cancer. 2007 Nov 1.

[17] Box NF. The role of p53 in pigmentation, tanning and melanoma. 2008 Oct.

[18] Hayward N. New developments in melanoma genetics. 2000 Jul. 
[19] Meyle KD. Genetic risk factors for melanoma. 2009 Oct.

[20] Soengas MS. Apoptosis and melanoma chemoresistance. 2003 May 19.

[21] Wang X, Jiang X. Mdm2 and MdmX partner to regulate p53. FEBS Lett 2012 May 21;586(10):1390-6.

[22] Ji Z. p53 rescue through HDM2 antagonism suppresses melanoma growth and potentiates MEK inhibition. 2012 Feb.

[23] Polsky D, Bastian BC, Hazan C, Melzer K, Pack J, Houghton A, et al. HDM2 protein overexpression, but not gene amplification, is related to tumorigenesis of cutaneous melanoma. Cancer Res 2001 Oct 15;61(20):7642-6.

[24] Pho L. Melanoma genetics: a review of genetic factors and clinical phenotypes in familial melanoma. 2006 Mar.

[25] Satyamoorthy K. p16INK4A and familial melanoma. 2003.

[26] Rayess H, Wang MB, Srivatsan ES. Cellular senescence and tumor suppressor gene p16. Int J Cancer 2012 Apr 15;130(8):1715-25.

[27] Sharpless E. The INK4a/ARF locus and melanoma. 2003 May 19.

[28] Meyle KD. Genetic risk factors for melanoma. 2009 Oct.

[29] Hayward N. New developments in melanoma genetics. 2000 Jul.

[30] Dahl C, Guldberg P. The genome and epigenome of malignant melanoma. APMIS 2007 Oct;115(10):1161-76.

[31] Anichini A. APAF-1 signaling in human melanoma. 2006 Jul 18.

[32] Anichini A. APAF-1 signaling in human melanoma. 2006 Jul 18.

[33] Mustika R. Decreased expression of Apaf-1 with progression of melanoma. 2005 Feb.

[34] Gray-Schopfer V, Wellbrock C, Marais R. Melanoma biology and new targeted therapy. Nature 2007 Feb 22;445(7130):851-7.

[35] Hussein MR, Haemel AK, Wood GS. Apoptosis and melanoma: molecular mechanisms. J Pathol 2003 Mar;199(3):275-88.

[36] Pustisek N, Situm M. UV-radiation, apoptosis and skin. Coll Antropol 2011 Sep;35 Suppl 2:339-41.

[37] Nakano K. PUMA, a novel proapoptotic gene, is induced by p53. 2001 Mar.

[38] Zinkel S, Gross A, Yang E. BCL2 family in DNA damage and cell cycle control. Cell Death Differ 2006 Aug;13(8):1351-9. 
[39] Yang G, Zhang G, Pittelkow MR, Ramoni M, Tsao H. Expression profiling of UVB response in melanocytes identifies a set of p53-target genes. J Invest Dermatol 2006 Nov;126(11):2490-506.

[40] Karst AM. PUMA expression is significantly reduced in human cutaneous melanomas. 2005 Feb 3.

[41] Bisso A. p73 as a pharmaceutical target for cancer therapy. 2011.

[42] Conforti F, Sayan AE, Sreekumar R, Sayan BS. Regulation of p73 activity by posttranslational modifications. Cell Death Dis 2012;3:e285.

[43] Johnson J, Lagowski J, Sundberg A, Kulesz-Martin M. P53 family activities in development and cancer: relationship to melanocyte and keratinocyte carcinogenesis. J Invest Dermatol 2005 Nov;125(5):857-64.

[44] Chen F, Wang W, El-Deiry WS. Current strategies to target p53 in cancer. Biochem Pharmacol 2010 Sep 1;80(5):724-30.

[45] de LJ, Ly LV, Lodder K, Verlaan-de VM, Teunisse AF, Jager MJ, et al. Synergistic growth inhibition based on small-molecule p53 activation as treatment for intraocular melanoma. Oncogene 2012 Mar 1;31(9):1105-16.

[46] Sauter ER, Takemoto R, Litwin S, Herlyn M. p53 alone or in combination with antisense cyclin D1 induces apoptosis and reduces tumor size in human melanoma. Cancer Gene Ther 2002 Oct;9(10):807-12.

[47] Bedikian AY, Millward M, Pehamberger H, Conry R, Gore M, Trefzer U, et al. Bcl-2 antisense (oblimersen sodium) plus dacarbazine in patients with advanced melanoma: the Oblimersen Melanoma Study Group. J Clin Oncol 2006 Oct 10;24(29):4738-45. 
